\title{
Quantum time machine
}

\author{
Pedro F. González-Díaz \\ Centro de Física “Miguel Catalán,”, Instituto de Matemáticas y Física Fundamental, Consejo Superior de Investigaciones Científicas, \\ Serrano 121, 28006 Madrid, Spain
}

(Received 8 December 1997; published 16 November 1998)

\begin{abstract}
The continuation of Misner space into the Euclidean region is seen to imply the topological restriction that the period of the closed spatial direction becomes time dependent. This restriction results in a modified Lorentzian Misner space in which the renormalized stress-energy tensor for quantized complex massless scalar fields becomes regular everywhere, even on the chronology horizon. A quantum-mechanically stable time machine with just the submicroscopic size may then be constructed out of the modified Misner space, for which the semiclassical Hawking chronology protection conjecture is no longer an obstruction.
\end{abstract}

[S0556-2821(98)00624-9]

PACS number(s): 04.20.Gz, 04.62.+v

\section{INTRODUCTION}

After the seminal papers by Morris, Thorne and Yurtsever [1], the notion of a time machine has jumped from the pages of science fiction books to those of scientific journals, giving rise to a recent influx of papers [2-7] and books [8,9] on the subject. Several potentially useful models for time machines have since been proposed, including the wormhole of Thorne et al. [1], Gott's couple of cosmic strings set at relative motion [2], Grant's extension [3], Politzer's machine [4], Roman rings [5] and, ultimately, a time machine based on the so-called ringholes [6].

Apart from all those difficulties related with potential violation of classical causality that would arise while travelling backward in time [8], all of these models show at least one of the two following shortcomings: an induced violation of the classical energy conditions for the matter allowing closed timelike curves (CTC's) [9] and, more importantly, the quantum instabilities originating from the divergences of the stress-energy tensor of vacuum fluctuations on the chronology horizon [8] (that is the onset of the nonchronal region where CTC's develop).

Violation of energy conditions is generally produced by the large inward pressure required on the tunnels and leads to the emergence of regions where matter has negative-energy density. This could no longer be a real problem as negative energy has become commonplace in quantum and semiclassical gravity [10,11]. More severe is the difficulty related with the need of having a tachyonic center of mass in parallel-moving cosmic strings which are subject, moreover, to unphysical boundary conditions [12].

By far the acutest problem with most of the proposed time machines is the emergence of the stress-energy divergences of the quantum vacuum fluctuations. This makes instable the spacetime construct and has actually led to the formulation of a chronology protection conjecture [13], according to which the laws of physics should prevent time travel to occur. So far, in spite of some attempts intended to violate it $[14,15]$, this conjecture has survived rather forcefully [16]. However, the realm where chronology protection holds is semiclassical physics, as it is for all hitherto proposed time machines. Actually, because spacetime foam [17] must entail strong violations of causal locality everywhere, one would expect the Hawking conjecture to be inapplicable in the framework of quantum gravity proper, and that the divergences of stress-energy tensor due to quantum vacuum fluctuations merely are artifacts from inappropriate combination of quantum matter fields with classical spacetime devices.

In this paper we consider an attempt to get rid of these divergences in a nonchronal spacetime constructed out from Misner space [18], where the thermal properties of Euclidean gravity are taken into account. The distinguishing feature of the considered spacetime with respect to usual Misner space is that the period of the closed spatial direction becomes now time-dependent, and the nonchronal region can only be acceded on if the time coordinate is quantized according to a pattern which is the same as that for the quantization being currently assumed [19] for the event horizon of black holes. We have checked that the stress-energy tensor for quantum vacuum fluctuations in this spacetime is regular everywhere, but only time machines with submicroscopic size can be constructed out of it.

The paper is organized as follows. In Sec. II we consider the continuation of Misner space into the Euclidean sector where the periodicity of the distinct spacetime directions are fixed and then continued back to the Lorentzian region, resulting in a modified structure for Misner space. The Hadamard function and hence the expectation value of the stressenergy tensor for quantum vacuum fluctuations are evaluated in Sec. III in the case of a complex massless scalar field propagating in the modified Misner space. It is checked that these quantities are regular everywhere. We conclude in Sec. IV, where the concept of a quantized time machine is also discussed. Throughout the paper units are used such that $c$ $=\hbar=G=1$.

\section{THE MODIFIED MISNER SPACE}

It has been stressed [20] that Misner space encompasses many remarkable pathologies in the structure of spacetime, including those of wormholes and ringholes and other spacetimes that contain potentially interesting time machines. Therefore, the spacetime structure and topology of the Misner space itself remain the focus of current attention. It is 
known that the metric of Misner space can be written as [21]

$$
d s^{2}=-d t^{2}+t^{2}\left(d x^{1}\right)^{2}+\left(d x^{2}\right)^{2}+\left(d x^{3}\right)^{2},
$$

where $0<t<\infty, 0 \leqslant x^{2}, x^{3} \leqslant \infty$, and $0 \leqslant x^{1} \leqslant 2 \pi$. With respect to Minkowski space, the fact that $x^{1}$ is periodic is the unique distinguishing property originating from its topology $S^{1} \times R^{3}$. Otherwise, they are identical. This can be seen by introducing the covering space through the coordinate transformations

$$
y^{0}=t \cosh \left(x^{1}\right), \quad y^{0}=t \sinh \left(x^{1}\right), \quad y^{2}=x^{2}, \quad y^{3}=x^{3},
$$

where the metric becomes

$$
d s^{2}=-\left(d y^{0}\right)^{2}+\left(d y^{1}\right)^{2}+\left(d y^{2}\right)^{2}+\left(d y^{3}\right)^{2} .
$$

If the period associated with direction $x^{1}$ is $a$, then the topology of the Misner space implies the identification of points on the covering space given by

$$
\begin{aligned}
& \left(y^{0}, y^{1}, y^{2}, y^{3}\right) \leftrightarrow\left(y^{0} \cosh (n a)+y^{1} \sinh (n a), y^{0} \sinh (n a)\right. \\
& \left.+y^{1} \cosh (n a), y^{2}, y^{3}\right),
\end{aligned}
$$

which corresponds to the identifications $\left(t, x^{1}, x^{2}, x^{3}\right)$ $\leftrightarrow\left(t, x^{1}+n a, x^{2}, x^{3}\right)$ in Misner coordinates.

Let us now consider the Euclidean continuation of metric (2.1). This will allow us to investigate how periodical properties of the distinct space directions in the Euclidean sector transform when they are rotated back to the Lorentzian region. Metric (2.1) becomes definite positive if we introduce the rotation

$$
t=i \tau, \quad x^{1}=i \chi
$$

Then,

$$
d s^{2}=d \tau^{2}+\tau^{2}(d \chi)^{2}+\left(d x^{2}\right)^{2}+\left(d x^{3}\right)^{2}
$$

An interesting property of the Euclideanized Misner space is that its covering space preserves the Lorentzian signature of the Minkowskian covering for the original Misner space. From Eqs. (2.2) and (2.5) we now have the coordinate transformations:

$$
\tilde{y}^{0}=i \tau \cos \chi, \quad \tilde{y}^{1}=-\tau \sin \chi, \quad \tilde{y}^{2}=x^{2}, \quad \tilde{y}^{3}=x^{3},
$$

so that

$$
d s^{2}=-\left(d \tilde{y}^{0}\right)^{2}+\left(d \tilde{y}^{1}\right)^{2}+\left(d \tilde{y}^{2}\right)^{2}+\left(d \tilde{y}^{3}\right)^{2} .
$$

However, the topology that corresponds to Euclideanized Misner space is different from what is associated with Eq. (2.3). For Eq. (2.8), the topology is $S^{1} \times S^{1} \times R^{2}$. This can be seen by noting that in the coordinates of metric (2.1), this metric has an apparent singularity at $t=0$. One can still extend metric (2.1) beyond $t=0$ by using new coordinates defined by [21]

$$
T=t^{2}, \quad V=\ln t+x^{1},
$$

with which metric (2.1) transforms into

$$
d s^{2}=-d V d T+T d V^{2}+\left(d x^{2}\right)^{2}+\left(d x^{3}\right)^{2} .
$$

In order to investigate the periodic properties of the directions in the Euclideanized Misner space, one can now introduce the extended coordinate definitions of the Lorentzian Misner extended metric

$$
u+w=V, \quad u-w=T-\int T d V,
$$

with which Eq. (2.10) becomes

$$
d s^{2}=-d u^{2}+d w^{2}+\left(d x^{2}\right)^{2}+\left(d x^{3}\right)^{2}
$$

and

$$
u^{2}-w^{2}=V\left(T-\int T d V\right), \quad \frac{u-w}{u+w}=\frac{T-\int T d V}{V} .
$$

Metric (2.12) will become positive definite provided we allow the continuation $u=i \zeta$; hence we have $\zeta^{2}+w^{2}$ $=V\left(-T+\int T d V\right)$ and, from Eq. (2.9), it can be seen that the section on which $\zeta$ and $w$ are both real will correspond to $t$ $\geqslant e^{1 / 2}, x^{1} \geqslant \frac{1}{2}$. That section defines a Misner instanton describing transitions on the chronal region. Using then Eq. (2.5) and the second of Eq. (2.13) one can finally write

$$
\begin{aligned}
\exp \left(\frac{i \zeta}{2 w}\right)= & \exp \left(\frac{\zeta^{2}-w^{2}}{2 w^{2}}\right) \tau^{\tau^{-2}} \exp \left(-\frac{2 \chi\left(\frac{\pi}{2}+\chi\right)}{\tau^{2}}\right) \\
& \times \exp \left[i\left(\frac{\pi / 2+\chi}{\tau^{2}}\right)\right]
\end{aligned}
$$

It follows that on the Euclidean sector, both $\tau$ and $\chi$ are periodic, with respective periods

$$
\Pi_{\tau}=\frac{1}{2}, \quad \Pi_{\chi}=2 \pi \tau^{2}=g(\tau)
$$

Therefore, the topology of the Euclidean Misner space is $S^{1} \times S^{1} \times R^{2}$, and one should expect that an observer in Misner space would detect a thermal bath at temperature $T_{M}$ $=2$.

Rotating back to the Lorentzian sector (i.e., taking $\tau=$ $-i t$ and $\chi=-i x^{1}$ ) we see that $t$ no longer becomes periodic, though $x^{1}$ still keeps a periodic character, with a period which should be given by

$$
a=|g(t)|=2 \pi t^{2} .
$$

Thus, the continuation to the Euclidean section of Misner space shows that the Lorentzian Misner space itself must be modified in that the period of coordinate $x^{1}$ should no longer be an arbitrary constant, but it depends on time $t$ according to Eq. (2.15). This change manifests in that the extension of the 
region covered by metric (2.1) across the chronology boundary $t=0$ into a new region that contains CTC's becomes modified in that the twisted null geodesics [21] spiral around and around now each time with a larger frequency as they approach $t=0$, to finally diverge at this point. On the Minkowskian covering space this modification translates as a concentration onto a single point of all the identified points on the surface $\sigma=\left(y^{0}\right)^{2}-\left(y^{1}\right)^{2}=0$ of the left and right chronology horizons. Actually, the need of making period $a$ proportional to $t^{2}$ arises from the kind of instantonic quantization implied by continuing into the Euclidean sector, so that the modified Misner space can be regarded as essentially semiclassical.

Removal of the resulting singularity at $t=0$ can only be achieved if we let time $t$ be quantized so that either (i) the point at $t=0$ is replaced by a minimum nonzero throat (i.e., by transforming $t$ according to $t^{2} \rightarrow t^{2}+R_{0}^{2}$, with $R_{0}^{2}$ a given small constant which defines the throat), so restoring the usual Misner space situation where twisted null geodesics terminated at $t=0$, leading to the existence of two inequivalent geodesically incomplete analytic extensions of Eq. (2.1), $V_{ \pm}= \pm \ln t+x^{1}$, which are locally inextendible [21], or (ii), if we want to establish full equivalence between the two possible analytic extensions $V_{ \pm}$(so that all null geodesics do not terminate at $t=0$ but are extendible beyond it), then time $t$ must be quantized in such a way that though the two extensions cover the whole space only some particular values of time are allowed.

A simple ansatz that implements this requirement is

$$
t^{2}=(n+\beta) C,
$$

where $n=0,1,2,3, \ldots, \beta$ is an arbitrary parameter of order unity, and $C$ defines again a throat at $n=0$. In the next section we shall see that it is such an ansatz that is actually required if we want a scalar field propagating in the modified Misner space to be expandible in an orthonormal basis with time-independent frequency.

Alternatively, one could have also rewritten Eq. (2.14) in the form:

$$
\exp (i \zeta)=i \exp \left(\frac{\zeta^{2}-w^{2}}{w}\right) \tau \exp \left(-2 \chi\left(\frac{\pi}{2}+\chi\right)\right) \exp (i \chi)
$$

If we would choose this ansatz, then only the coordinate $\chi$ would turn out to be periodic with a period $2 \pi$, while the Euclidean time $\tau$ became no longer periodic. Rotating back to the Lorentzian sector, we then had $a=2 \pi$, a result which has already been obtained by Cassidy [22] and by $\mathrm{Li}$ and Gott [23]. The latter authors have shown that, associated to $a=2 \pi$, there must be a self-consistent vacuum for Misner space for which the renormalized stress-energy tensor is zero throughout the Misner space. We do not consider this choice to be physically allowable, however, because it leads to a nonperiodic Euclidean time $\tau$ and hence to a Misner space without any background thermal radiation, in spite of the feature that Misner space has an apparent horizon at $t=0$.

\section{THE HADAMARD FUNCTION}

In what follows we shall consider the propagation of a complex scalar field $\phi$ in the modified Misner space discussed in Sec. II. For the sake of generality we will require $\phi$ to satisfy the automorphic condition [24] $\left(X \equiv t, x^{1}, x^{2}, x^{3}\right)$

$$
\phi(\gamma X)=e^{2 \pi i \alpha} \phi(X), \quad 0 \leqslant \alpha \leqslant \frac{1}{2},
$$

with $\alpha$ the automorphic parameter and $\gamma$ representing the symmetry (periodicity identifications) transformation of the modified Misner space; i.e., the transformation will be taken to satisfy the periodicity condition for a period $2 \pi t^{2}$. We shall follow the analysis carried out by Sushkov [14] and look at complex scalar fields that obey the field equation

$$
\square \phi=\square \bar{\phi}=0 .
$$

On the covering space coordinates, this equation admits the general positive-frequency solution:

$$
\begin{aligned}
\phi\left(y^{0}, y^{1}, y^{2}, y^{3}\right)= & \int d k_{1} d k_{2} d k_{3} \frac{A\left(k_{0}, k_{1}, k_{2}, k_{3}\right)}{4 \pi^{3 / 2} k_{0}} \\
& \times e^{i\left(-k_{0} y^{0}+k_{1} y^{1}+k_{2} y^{2}+k_{3} y^{3}\right)},
\end{aligned}
$$

where $k_{0}=\sqrt{k_{1}^{2}+k_{2}^{2}+k_{3}^{2}}$ and $A\left(k_{0}, k_{1}, k_{2}, k_{3}\right)$ is a given spectral function.

The demand that $\phi$ satisfies condition (3.1) amounts to the functional relation

$$
\begin{aligned}
\phi\left(y^{0} \cosh \left(2 \pi t^{2}\right)+y^{1} \sinh \left(2 \pi t^{2}\right), y^{0} \sinh \left(2 \pi t^{2}\right)\right. \\
\left.\quad+y^{1} \cosh \left(2 \pi t^{2}\right), y^{2}, y^{3}\right) \\
=e^{2 \pi i \alpha} \phi\left(y^{0}, y^{1}, y^{2}, y^{3}\right),
\end{aligned}
$$

with which automorphicity for the solutions will hold provided

$$
\begin{aligned}
A\left(k_{0} \cosh \left(2 \pi t^{2}\right)-k_{1} \sinh \left(2 \pi t^{2}\right),-k_{0} \sinh \left(2 \pi t^{2}\right)\right. \\
\left.\quad+k_{1} \cosh \left(2 \pi t^{2}\right), k_{2}, k_{3}\right) \\
=e^{2 \pi i \alpha} A\left(k_{0}, k_{1}, k_{2}, k_{3}\right) .
\end{aligned}
$$

This admits a general solution which is formally the same as that obtained by Sushkov [14], i.e.,

$$
A\left(k_{0}, k_{1}, k_{2}, k_{3}\right)=\sum_{n=-\infty}^{\infty} C_{n}\left(k_{2}, k_{3}\right)\left(k_{0}-k_{1}\right)^{i \nu},
$$

[where again the $C_{n}\left(k_{2}, k_{3}\right)$ are arbitrary functions of $k_{2}$ and $k_{3}$ ], but in which the frequency $\nu$ would in principle have explicit dependence on time:

$$
\nu=-\frac{n+\alpha}{t^{2}} .
$$

Simple demand of time independence for frequency $\nu$ would in principle just imply that $t=$ const, but if one adds 
the requirement that the whole modified Misner space be taken into account for any variable values of $x^{1}$ and hence $t$, then the above demand of time independence for $\nu$ would imply quantization of time $t$ according to the law

$$
t^{2}= \pm(n+\alpha) t_{0}^{2}
$$

where $t_{0}^{2}$ is an arbitrary constant. Clearly, the quantity $t^{2}$ can also take on negative values. This quantal result would be no surprise. When one deals with a spacetime which admits CTC's that can travel into the past, negative time can be given an interpretation, much as one can do with, e.g., the negative eigenenergy solutions of Dirac equation. Anyway, if one wants to guarantee positiveness for $t^{2}$, one can always associate the plus sign in Eq. (3.7) with the positive integer values $n=0,1,2, \ldots$, and the minus sign in Eq. (3.7) with the negative integer values $n=-1,-2,-3, \ldots$. This quantization condition for time $t$ is exactly of the same form as that of the ansatz used in Sec. II in order to avoid the singularity at $t=0$, and leads finally to a frequency given by $\nu=$ $\mp t_{0}^{-2}$.

Inserting Eq. (3.5) into Eq. (3.2) and first carrying out the integration over $k_{1}$ and then transforming to Misner coordinates [14], we obtain finally for the automorphic field

$$
\phi\left(t, x^{1}, x^{2}, x^{3}\right)=\sum_{n=0}^{\infty} \int d k_{2} \int d k_{3} \widetilde{C}_{n}\left(k_{2}, k_{3}\right) \varphi\left(t, x^{1}, x^{2}, x^{3}\right),
$$

where

$$
\varphi\left(t, x^{1}, x^{2}, x^{3}\right)=D(n) H_{-i t_{0}^{-2}}^{(2)}(k t) e^{i\left(-t_{0}^{-2} x^{1}+k_{2} x^{2}+k_{3} x^{3}\right)},
$$

with $H_{i \nu}^{(2)}(k t)$ the Bessel function of the third kind, $D(n)$ a normalizing coefficient, and $k=\sqrt{k_{2}^{2}+k_{3}^{2}}$. Adopting the definition of the scalar product of Ref. [14], one can now show that the solutions (3.9) form an orthonormal basis in a Hilbert space provided we choose

$$
D(n)=\frac{\sqrt{2}}{8 \pi t e^{\pi(n+\alpha) / 2 t^{2}}}=\frac{\sqrt{2}}{8 \pi \sqrt{n+\alpha} t_{0} e^{\pi / 2 t_{0}^{2}}} .
$$

Noting that for two generally different sets of Misner coordinates $\left(X \equiv\left\{x^{i}\right\}\right.$ and $\left.\widetilde{X} \equiv\left\{\tilde{x}^{i}\right\}, \quad i=0,1,2,3\right)$ the corresponding arbitrary frequencies $\nu$ and $\tilde{\nu}$ are the same, we can finally calculate the renormalized Hadamard function. This will be taken to be given by $(\mu, \nu=0,1,2,3, \beta, \gamma=1,2,3)$

$$
\begin{aligned}
G_{r e n}^{(1)}\left(X^{\beta}, \widetilde{X}^{\gamma}\right) & =\left.\sum_{\tilde{n}=0}^{\infty} G_{r e n}^{(1)}\left(X^{\mu}, \widetilde{X}^{\nu}\right)\right|_{t=\sqrt{n+\alpha} t_{0}, \tilde{t}=\sqrt{\tilde{n}+\alpha} t_{0}} \\
& =\left.\left(\sum_{n=0}^{\infty} \sum_{\tilde{n}=0}^{\infty} \iint d k_{2} d k_{3}\left[\varphi\left(X^{\mu}\right) \bar{\varphi}\left(\tilde{X}^{\nu}\right)+\varphi\left(\tilde{X}^{\mu}\right) \bar{\varphi}\left(X^{\nu}\right)\right]-G_{0}^{(1)}\left(X^{\mu}, \widetilde{X}^{\nu}\right)\right)\right|_{t=\sqrt{n+\alpha} t_{0}, \tilde{t}=\sqrt{\tilde{n}+\alpha} t_{0}} \\
& =\left.\frac{1}{4 \pi^{2}}\left(\frac{1}{\sigma\left(X^{\mu}, \tilde{X}^{\nu}\right)}-\frac{1}{\sigma_{0}\left(X^{\mu}, \tilde{X}^{\nu}\right)}\right)\right|_{t=\tilde{t}=\sqrt{\alpha} t_{0}}+\left.\frac{1}{\pi^{2}} \sum_{n=1}^{\infty} \sum_{\tilde{n}=1}^{\infty} \frac{\cosh \left[n\left(x^{1}-\tilde{x}^{1}\right)\right] \sin (n \arccos \chi)}{t \tilde{t} \sqrt{1-\eta^{2}}} \Psi_{n}(\alpha, t)\right|_{A},
\end{aligned}
$$

where the subscript $A$ means evaluation at

$$
A\left\{t=\sqrt{n+\alpha} t_{0}, \tilde{t}=\sqrt{\tilde{n}+\alpha} t_{0}\right\}
$$

$G_{r e n}^{(1)}\left(X^{\mu}, \widetilde{X}^{\nu}\right)$ is the Hadamard function obtained in Ref. [14], with $\eta$ defined as $\chi$ in [14] and $\Psi_{n}(\alpha, t)$ given now by

$$
\Psi_{n}(\alpha, t)=\frac{e^{2 \pi n t^{2}} \cos (2 \pi \alpha)-1}{e^{4 \pi n t^{2}}-2 e^{2 \pi n t^{2}} \cos (2 \pi \alpha)+1}
$$

and

$$
\begin{gathered}
\sigma\left(X^{\mu}, \tilde{X}^{\nu}\right)=\frac{1}{2}\left\{-(t-\tilde{t})^{2}+2 t \tilde{t}\left[\cosh \left(x^{1}-\tilde{x}^{1}\right)-1\right]+\left(x^{2}-\tilde{x}^{2}\right)^{2}+\left(x^{3}-\tilde{x}^{3}\right)^{2}\right\} \\
\sigma_{0}\left(X^{\mu}, \tilde{X}^{\nu}\right)=\frac{1}{2}\left\{-(t-\tilde{t})^{2}+t^{2}\left(x^{1}-\tilde{x}^{1}\right)+\left(x^{2}-\tilde{x}^{2}\right)^{2}+\left(x^{3}-\tilde{x}^{3}\right)^{2}\right\}
\end{gathered}
$$

The final result of this calculation is 


$$
G_{r e n}^{(1)}\left(X^{\beta}, \tilde{X}^{\gamma}\right)=\frac{1}{4 \pi^{2}}\left(\frac{1}{\sigma\left(X^{\beta}, \tilde{X}^{\gamma}\right)}-\frac{1}{\sigma_{0}\left(X^{\beta}, \tilde{X}^{\gamma}\right)}\right)+\frac{1}{\pi^{2}} \sum_{n=1}^{\infty} \sum_{\tilde{n}=1}^{\infty} \frac{\cosh \left[n\left(x^{1}-\tilde{x}^{1}\right)\right] \sin (n \arccos \chi)}{\sqrt{n+\alpha} \sqrt{\tilde{n}+\alpha} t_{0}^{2} \sqrt{1-\eta^{2}}} \Psi_{n}(\alpha),
$$

where

$$
\begin{gathered}
\Psi_{n}(\alpha)=\frac{e^{2 \pi n(n+\alpha) t_{0}^{2}} \cos (2 \pi \alpha)-1}{e^{4 \pi n(n+\alpha) t_{0}^{2}}-2 e^{2 \pi n(n+\alpha) t_{0}^{2}} \cos (2 \pi \alpha)+1}, \\
\sigma\left(X^{\beta}, \widetilde{X}^{\gamma}\right)=\frac{1}{2}\left\{2 \alpha t_{0}^{2}\left[\cosh \left(x^{1}-\tilde{x}^{1}\right)-1\right]+\left(x^{2}-\tilde{x}^{2}\right)^{2}+\left(x^{3}-\tilde{x}^{3}\right)^{2}\right\}, \\
\sigma_{0}\left(X^{\beta}, \widetilde{X}^{\gamma}\right)=\frac{1}{2}\left\{\alpha t_{0}^{2}\left(x^{1}-\tilde{x}^{1}\right)+\left(x^{2}-\tilde{x}^{2}\right)^{2}+\left(x^{3}-\tilde{x}^{3}\right)^{2}\right\} .
\end{gathered}
$$

It follows that the value of $G_{r e n}^{(1)}\left(X^{\beta}, \widetilde{X}^{\gamma}\right)$ remains finite on the whole modified Misner space for any value of $\alpha$, even $\alpha$ $=0$, provided the arbitrary constant $t_{0}$ is nonzero. This result immediately reflects in the remarkable implication that the renormalized vacuum expectation values of the scalar field squared, $\left\langle\phi^{2}\right\rangle=\left\langle 0\left|\phi^{2}\right| 0\right\rangle$, and stress-energy tensor, $\left\langle T_{\mu \nu}\right\rangle$ $=\left\langle 0\left|T_{\mu \nu}\right| 0\right\rangle$, remain both finite as well on the nonsingular modified Misner space. These quantities are evaluated as follows:

$$
\begin{gathered}
\left\langle\phi^{2}\right\rangle=\lim _{\tilde{X} \rightarrow X} G_{r e n}^{(1)}\left(X^{\beta}, \widetilde{X}^{\gamma}\right)=\frac{1}{\pi^{2} t_{0}^{2}} \sum_{n=1}^{\infty} \frac{n \Psi(\alpha)}{n+\alpha}, \\
\left\langle T_{\beta \gamma}\right\rangle=\lim _{\tilde{X} \rightarrow X}\left\{\left.\left[(1-\xi) \nabla_{\mu} \widetilde{\nabla}_{\nu}+\left(2 \xi-\frac{1}{2}\right) g_{\mu \nu} \nabla_{\alpha} \widetilde{\nabla}^{\alpha}-2 \xi \nabla_{\mu} \nabla_{\nu}+\frac{1}{2} \xi g_{\mu \nu} \square\right] G_{r e n}^{(1)}\left(X^{\mu}, \widetilde{X}^{\nu}\right)\right|_{\left.t=\sqrt{n+\alpha} t_{0}, \tilde{t}=\sqrt{\tilde{n}+\alpha} t_{0}\right\}}\right\} \\
=\frac{1}{\pi^{2} t_{0}^{4}} \sum_{n=1}^{\infty} \frac{\operatorname{diag}(L, 3 L, M, M)}{(n+\alpha)^{2}}+\frac{1}{\pi^{2}} F\left(\alpha, t_{0}\right),
\end{gathered}
$$

where $F\left(\alpha, t_{0}\right)$ is a complicated function of $\alpha$ and $\xi$ arising from the time derivatives of the function $\Psi_{n}(\alpha, t)$, which is regular for all values of $\alpha$ and $\xi$; $\xi$ is the coupling parameter for the scalar field ( $\xi=0$ for minimal coupling and $\xi=\frac{1}{6}$ for conformal coupling) and $L$ and $M$ are as given in Ref. [14], but with $\Psi_{n}(\alpha, a)$ replaced for $\Psi_{n}(\alpha)$, as given in Eq. (3.16), that is

$$
\begin{gathered}
L=\frac{1}{\pi^{2}} \sum_{n=1}^{\infty}\left(\frac{n-n^{3}}{3}-\frac{3 \xi n}{2}\right) \Psi_{n}(\alpha) \\
M=\frac{1}{\pi^{2}} \sum_{n=1}^{\infty}\left(\frac{2 n+n^{3}}{3}-\frac{9 \xi n}{2}\right) \Psi_{n}(\alpha) .
\end{gathered}
$$

Of course, all the above calculations are nonstrict with the mathematical point of view, as we have dealt with solutions of a field equation, while considering the time $t$ as a discrete quantity. Our manipulations should therefore be regarded to be rather a qualitative procedure by means of which quantum-gravity effects are taken into account in the semiclassical field solutions by introducing discrete values of time. As shown in Eqs. (3.11), (3.19) and (3.20), this manifests in the fact that both the Hadamard function and the square of the field and the renormalized stress-energy tensor can all be obtained immediately from the corresponding expressions in Ref. [14] by restricting time to satisfy Eq. (3.7) for the positive case, and $\nu=-t_{0}^{2}$.

For the particular case of nontwisted fields, $\alpha=0$, expression (3.12) reduces to

$$
\Psi\left(t_{0}\right)=\left(e^{2 \pi n^{2} t_{0}^{2}}-1\right)^{-1},
$$

so that the quantities $\left\langle\phi^{2}\right\rangle$ and $\left\langle T_{\beta \gamma}\right\rangle$ are finite even when $\alpha=0$. Clearly, one always could renormalize the stressenergy tensor further, so that it becomes $\left\langle T_{\beta \gamma}\right\rangle=0$ everywhere.

Thus, all the divergences coming from quantum vacuum fluctuations that take place on the chronology horizon of the classical Misner space whose periodic direction has constant period are smoothed out on the modified, no longer classical Misner space where the period along the periodic direction is time dependent, for which case the expectation values of the scalar field squared and stress-energy tensor are regular everywhere for any value of $\alpha$ and $\xi$, provided $t_{0} \neq 0$.

\section{CONCLUSIONS: THE QUANTUM TIME MACHINE}

In this paper we have considered the change of topology implied by the continuation from Lorentzian Misner space 
into its Euclidean counterpart; i.e., from $S^{1} \times R^{3}$ to $S^{1} \times S^{1}$ $\times R^{2}$. It was seen that the spatial direction which is also periodic in the Euclidean continuation has a period that depends on the Euclidean time and rotates back to an also time-dependent period on the Lorentzian sector. This induces a change in the structure of the Misner space, giving rise to a true singularity at $t=0$, instead of the apparent singularity on the chronology horizon. A chronology horizon with nonzero width can only be restored if time is quantized according to a rule which parallels that assumed for the quantization of the black hole event horizon [19].

We can show that the region $t<0$ of the modified Misner space keeps its nonchronal character everywhere only if $\alpha t_{0}^{2}<1$, that is in the quantum-gravity regime. Consider the line $L_{1}$ defined by $t=-\omega x^{1}, x^{2}=x^{3}=0$, which will be timelike everywhere provided $2 \pi \omega<1$, and the circle $L_{0}$ defined by $t=\sqrt{\alpha} t_{0}>0, x^{2}=x^{3}=0$, which is also timelike everywhere if $\sqrt{\alpha} t_{0}<1$. It is then easy to see that on $L_{1}$, the point $Q\left(x^{1}=\sqrt{\alpha} / \omega\right)$ precedes the point $P\left(x^{1}=2 \pi\right.$ $+\sqrt{\alpha} / \omega)$, but these two points are also on circle $L_{0}$ where $P$ precedes $Q$, provided $\omega>0$. Therefore, there will be CTC's always that the condition

$$
1>2 \pi \omega \geqslant \sqrt{\alpha} t_{0},
$$

holds, and hence the above conclusion follows.

The modified Misner space could be therefore transformed into a time machine by simply replacing the identified flat planes for identified spheres or tori, and allowing one of the resulting spherical or toroidal mouths to move relative to the respective other mouth. This would be equivalent to extract two spheres or tori from three-dimensional Euclidean space and identify the sphere or torus surfaces, while they are set in relative motion, so when you enter the surface of one you find yourself emerging from the surface of the other. In Minkowski spacetime, the time machine is obtained identifying the two world tubes swept out by the approaching (or receding) spheres or tori, with events at the same Lorentz time identified. The result would be what one may call quantum time machine. The chronology horizon of this construct is not on just the single surface at $t=0$, but is spread throughout a time strip with nonzero width, around the surface at $t=0$.

We have also checked that the quantum vacuum polarization has no catastrophic effects anywhere in the modified Misner space, so quantum time machines are quantummechanically stable. One would moreover expect that when their size approach the Planck length scale, these machines are spontaneously created in the quantum-gravity framework of the spacetime foam [17]. We do not know the probability for the existence of such machines, but the very definition of the foam requires violation of causal locality everywhere, and hence one can assume the existence of submicroscopic quantum time machines as a pre-requisite for the existence of the spacetime foam itself, and may therefore ascribe a probability of order unity for the existence of time machines in the foam.

The possibility that a future technology [1], or present natural process taking place somewhere in the universe [25], be able to pull a quantum time machine with extremely large spacetime curvature out from the quantum spacetime foam, and then grow it up to macroscopic size [1], will largely reside on the probability of existence that the macroscopic machine may have. Such a probability may still be estimated by instantonic techniques in the semiclassical approximation. On the $\tau-\chi$ section of the instanton studied in Sec. II, any boundary has topology $S^{1} \times S^{1}$ and so is compact. Since the scalar curvature of that section vanishes, the action can then be written only in terms of the surface integrals corresponding to the fixed boundaries. For the case being considered, this action is

$$
I_{E} \simeq \frac{1}{8 \pi} \int_{\partial M} d x^{1}\left(K-K^{0}\right)=\frac{1}{8 \pi} \int_{0}^{a} d x^{1}=\frac{1}{4},
$$

where $K$ is the trace of the second fundamental form of the boundary and $K^{0}$ the same for the boundary embedded in flat space. Hence, the semiclassical probability, $P \simeq e^{-I_{E}}$, for the spontaneous creation of macroscopic time machines would be expected to be of order unity too.

The chronology protection conjecture advanced by Hawking [13] has an essential semiclassical character. Therefore, the feature that a quantum time machine has not quantum instabilities does not necessarily imply violation of the conjecture, but rather the need for a new one which would state that fully quantized laws of physics require the existence of stable time machines, at least in the framework of quantum gravity, since otherwise no spacetime foam could exist. Whether or not this conjecture would ultimately imply the possibility of technologically growing one of these submicroscopic time machines up to a macroscopic size that remains stable is a matter which our present knowledge does not allow us to decide on. Nevertheless, if we keep in the semiclassical regime where no quantization of time can be assumed, then the true singularity at $t=0$ of the modified Misner space will prevent access to the nonchronal region and, in at least this sense, Hawking's conjecture would continue to hold.

\section{ACKNOWLEDGMENTS}

For useful comments, the author thanks L. J. Garay and G. A. Mena Marugán of IMAFF. This research was supported by DGICYT under Research Projects No. PB94-0107 and, partially, No. PB93-0139.
[1] M. J. Morris, K. S. Thorne, and U. Yurtsever, Phys. Rev. Lett. 61, 1446 (1988); M. S. Morris and K. S. Thorne, Am. J. Phys. 56, 395 (1988).

[2] J. Gott, Phys. Rev. Lett. 66, 1126 (1991).
[3] J. D. E. Grant, Phys. Rev. D 47, 2388 (1993).

[4] H. D. Politzer, Phys. Rev. D 49, 3981 (1994).

[5] T. A. Roman, Phys. Rev. D 47, 1370 (1993).

[6] P. F. González-Díaz, Phys. Rev. D 54, 6122 (1996). 
[7] B. Jensen and H. H. Soleng, Phys. Rev. D 45, 3528 (1992).

[8] K. S. Thorne, Black Holes and Time Warps (Norton, New York, 1994).

[9] M. Visser, Lorentzian Wormholes (AIP, Woodbury, 1996).

[10] N. D. Birrell and P. C. W. Davies, Quantum Fields in Curved Space (Cambridge University Press, Cambridge, England, 1982).

[11] M. J. Spaarnay, Physica (Amsterdam) 24, 751 (1958); D. Tabor and R. H. S. Winterton, Proc. R. Soc. London A312, 435 (1969).

[12] S. Deser and A. R. Steif, in Directions in General Relativity. Vol. 1, edited by B. L. Hu, M. P. Ryan, Jr., and C. V. Vishveshwara (Cambridge University Press, Cambridge, England, 1993).

[13] S. W. Hawking, Phys. Rev. D 46, 603 (1992).

[14] S. V. Sushkov, Class. Quantum Grav. 14, 523 (1997).

[15] S. V. Krasnikov, Phys. Rev. D 54, 7322 (1996).
[16] B. S. Kay, M. J. Radzikowski, and R. M. Wald, Commun. Math. Phys. 183, 533 (1997).

[17] S. W. Hawking, Nucl. Phys. B144, 349 (1978).

[18] C. W. Misner, in Relativity Theory and Astrophysics I. Relativity and Cosmology, edited by J. Ehlers (American Mathematical Society, Providence, RI, 1967).

[19] J. D. Bekenstein, Lett. Nuovo Cimento 11, 467 (1974); "Quantum Black Holes as Atoms,"' gr-qc/9710076.

[20] K. S. Thorne, in Directions in General Relativity, edited by B. L. Hu, M. P. Ryan, Jr., and C. V. Vishveshwara (Cambridge University Press, Cambridge, England, 1993), Vol. 1.

[21] S. W. Hawking and G. F. R. Ellis, The Large Scale Structure of Space-time (Cambridge University Press, Cambridge, England, 1973).

[22] M. J. Cassidy, Class. Quantum Grav. 14, 3031 (1997).

[23] Li-Xin Li and J. R. Gott III, Phys. Rev. Lett. 80, 2980 (1998).

[24] R. Banach and J. S. Dowker, J. Phys. A 12, 2527 (1979).

[25] P. F. González-Díaz, Phys. Rev. D 56, 6293 (1997). 